\title{
ON THE SECTIONAL CURVATURE OF HOLOMORPHIC CURVATURE OPERATORS
}

\author{
IGNACIO GUERRERO AND STANLEY M. ZOLTEK
}

\begin{abstract}
The object of this paper is to study the pointwise behaviour of the holomorphic sectional curvature function of a Kähler manifold and its relationship with the Riemannian curvature tensor.

We begin by defining an orthogonal projection from the space of positive semidefinite Kähler curvature operators into the space of Kähler curvature operators which satisfy the Bianchi identity and have nonnegative holomorphic sectional curvature. In dimension four we show that this map is onto. As a corollary we obtain a description of the minimum and maximum sets of the holomorphic sectional curvature function.

For each higher dimension, we exhibit an example of a Kähler curvature operator which is not the projection of a positive semidefinite one. Specifically, we construct a Kähler curvature operator with nonnegative holomorphic sectional curvature, whose zero set does not admit a description as in dimension four. These examples contradict results in the literature which claim to describe the extremal sets for the holomorphic sectional curvature function for all dimensions.
\end{abstract}

Introduction. The object of this paper is to study the pointwise behaviour of the holomorphic sectional curvature function $\sigma$ of a Kähler manifold and its relationship with the Riemannian curvature tensor $R$. Even though $R$ and $\sigma$ determine each other, the relationship between them is not completely understood.

Since we are interested in the pointwise behaviour of $\sigma$ and $R$, our setting will be that of an arbitrary $2 n$-dimensional real vector space $V$ with a complex structure $J$ and a hermitian inner product. The automorphism $J$ induces a symmetric linear operator (also denoted by $J$ ) on $\Lambda^{2}(V)$. The Grassmann manifold $G$ of oriented 2-planes in $V$ can be embedded in a natural way in $\Lambda^{2}(V)$. We denote by $G^{J} \subseteq G$ the set of all planes invariant under $J$ (holomorphic planes). A Kähler curvature operator is a symmetric linear operator on $\Lambda^{2}(V)$ satisfying $R J=J R=R$. The holomorphic sectional curvature function is given by $\sigma_{R}(P)=\langle R P, P\rangle, P \in G^{J}$.

We begin by defining an orthogonal projection from the space of positive semidefinite Kähler curvature operators into the space of Kähler curvature operators which satisfy the Bianchi identity and have nonnegative holomorphic sectional curvature. For dimension $V=4$ we show that this map is onto. In the real case this result is due to Thorpe [4]. The proof given in this paper can be easily modified to give an alternate proof of Thorpe's theorem. As a corollary we obtain the description of the minimum and maximum sets of $\sigma$ as the intersection of $G^{J}$ with linear subspaces of $\Lambda^{2}(V)$. A similar description is given in Naveira and Fuertes [2].

Received by the editors December 23, 1980.

1980 Mathematics Subject Classification. Primary 53B35; Secondary 53C55, 32C10.

Key words and phrases. Kähler curvature operator, holomorphic sectional curvature. 
Naveira and Fuertes [2] appeared to have extended the description of the extremal sets of $\sigma$ to all dimensions. We show that these results do not hold for dimension $V=2 n, n \geqslant 3$. Specifically, for each $n, n \geqslant 3$, we exhibit a Kähler curvature operator with nonnegative holomorphic sectional curvature whose zero set is not the intersection with $G^{J}$ of a linear subspace of $\Lambda^{2}(V)$. Analogous examples, in the real case, have been constructed by Zoltek [5]. The examples given in this paper are motivated by those in [5]. However, nontrivial modifications were required. The proofs provided here are self-contained and do not depend on [5].

The authors wish to thank Professor R. Geroch for helpful conversations.

1. Preliminaries. Let $V$ be a $2 n$-dimensional real vector space with a complex structure automorphism $J$ and a hermitian inner product $\langle\rangle,(\langle J x, J y\rangle=\langle x, y\rangle$ for all $x, y \in V)$. The Grassmannian $G$ of oriented 2-planes in $V$ will be identified with the set of all decomposable vectors of length one in $\Lambda^{2}(V)$. The automorphism $J$ induces a symmetric linear operator on $\Lambda^{2}(V)$ by $J(x \wedge y)=J x \wedge J y$. An oriented 2-plane $P \in G$ is called holomorphic if $J P=P$. By $G^{J} \subseteq G$ we denote the set of all holomorphic planes. If $P$ is holomorphic and $x$ is a unit vector in $P$, then $\{x, J x\}$ is an orthonormal basis for $P$ and hence $P=x \wedge J x$.

A curvature operator on a finite-dimensional inner product space $V$ is a symmetric linear operator on $\Lambda^{2}(V)$. By $R$ we denote the space of all curvature operators with inner product given by $\langle R, T\rangle=\operatorname{trace} R T, R, T \in R$. The sectional curvature of $R$ is the real valued function $\sigma_{R}(P)=\langle R P, P\rangle, P \in G$. It is easily checked that the set of all curvature operators satisfying the standard first Bianchi identity forms a linear subspace of $\mathscr{R}$ which we denote by $\mathscr{B}$. Let $\mathcal{S}$ be the orthogonal complement of $\mathscr{B}$ in $\mathscr{R}$; thus $\mathscr{R}=\mathscr{B} \oplus \mathcal{S}$ where $\oplus$ means orthogonal direct sum. $\mathcal{S}$ is characterized by $S \in \mathcal{S}$ if and only if $\sigma_{S} \equiv 0$ (see Singer and Thorpe [3]).

Let $V$ be a hermitian inner product space with complex structure $J$. We saw that $J$ induces a curvature operator on $V$ (also denoted by $J$ ). $R \in R$ is called Kähler if $R J=J R=R$. Define $\mathscr{R}_{K}$ (resp. $\mathscr{B}_{K}$ ) as the intersection of $\mathscr{R}$ (resp. $\mathscr{B}$ ) with the subspace of Kähler curvature operators. Let $\delta_{K}$ be the orthogonal complement of $\mathscr{B}_{K}$ in $\Re_{K}$. We then have $\mathscr{R}_{K}=\mathscr{B}_{K} \oplus \mathfrak{S}_{K}$. Given $R \in \mathfrak{R}_{K}$ its holomorphic sectional curvature is the restriction of $\sigma_{R}$ (also denoted by $\sigma_{R}$ ) to $G^{J}$. The zero set of $\sigma_{R}$ (also called the zero set of $R$ ) is $Z(R)=\left\{P \in G^{J} \mid \sigma_{R}(P)=0\right\}$. The space $\delta_{K}$ is characterized by $S \in \mathcal{S}_{K}$ if and only if $\sigma_{S} \equiv 0$ on $G^{J}$ (see Johnson [1]).

We denote by $\Lambda^{2}(V)^{J}$ the space of all $\xi \in \Lambda^{2}(V)$ such that $J \xi=\xi$. Let $\left\{e_{1}, \ldots, e_{n}, e_{1^{*}}=J e_{1}, \ldots, e_{n^{*}}=J e_{n}\right\}$ be an orthonormal basis for $V$; then $\left\{e_{i} \wedge\right.$ $\left.e_{i^{*}},\left(e_{i} \wedge e_{j}+e_{i^{*}} \wedge e_{j^{*}}\right) / \sqrt{2},\left(e_{i} \wedge e_{j^{*}}-e_{i^{*}} \wedge e_{j}\right) / \sqrt{2} ; 1<i<j<n\right\}$ is an orthonormal basis for $\Lambda^{2}(V)^{J}$. The set of vectors $H=\left\{\left(e_{i} \wedge e_{j}-e_{i^{*}} \wedge e_{j^{*}}\right) / \sqrt{2}\right.$, $\left.\left(e_{i} \wedge e_{j^{*}}+e_{i^{*}} \wedge e_{j}\right) / \sqrt{2} ; 1 \leqslant i<j \leqslant n\right\}$ is an orthonormal basis for the orthogonal complement of $\Lambda^{2}(V)^{J}$. It is easily checked that $R$ is Kähler if and only if $H \subseteq \operatorname{Ker} R$. Hence $\Re_{K}$ can be identified with the space of symmetric linear operators on $\Lambda^{2}(V)^{J}$. Note also that $G^{J} \subseteq \Lambda^{2}(V)^{J}$.

Define a map $T: R \rightarrow \Re_{K}$ by $T(R)=\frac{1}{4}(R+J R+R J+J R J)$. 
Proposition. 1. $T$ is a projection of $\mathcal{R}$ onto $\Re_{K}$.

2. $\sigma_{R}(P)=\sigma_{T(R)}(P)$ for $P \in G^{J}$.

3. $\langle T(R), S\rangle=\langle R, T(S)\rangle$ for all $R, S \in R$.

4. $T$ maps $\mathcal{S}$ onto $\mathcal{S}_{K}$.

Proof. The verification of 1 and 2 is straightforward. To prove claim 3 note that

$$
\langle T(R), S\rangle=\operatorname{trace} T(R) S=\frac{1}{4} \operatorname{trace}(R S+J R S+R J S+J R J S) .
$$

Hence if $\left\{v_{1}, \ldots, v_{m}\right\}$ denotes an orthonormal basis for $\Lambda^{2}(V)$ then

$$
\begin{aligned}
\langle T(R), S\rangle & =\frac{1}{4} \sum_{i=1}^{m}\left[\left\langle R S v_{i}, v_{i}\right\rangle+\left\langle J R S v_{i}, v_{i}\right\rangle+\left\langle R J S v_{i}, v_{i}\right\rangle+\left\langle J R J S v_{i}, v_{i}\right\rangle\right] \\
& =\frac{1}{4} \sum_{i=1}^{m}\left[\left\langle R S v_{i}, v_{i}\right\rangle+\left\langle R S J J v_{i}, J v_{i}\right\rangle+\left\langle R J S v_{i}, v_{i}\right\rangle+\left\langle R J S J J v_{i}, J v_{i}\right\rangle\right] \\
& =\frac{1}{4} \sum_{i=1}^{m}\left[\left\langle R S v_{i}, v_{i}\right\rangle+\left\langle R S J v_{i}, v_{i}\right\rangle+\left\langle R J S v_{i}, v_{i}\right\rangle+\left\langle R J S J v_{i}, v_{i}\right\rangle\right] \\
& =\frac{1}{4} \operatorname{trace}(R S+R S J+R J S+R J S J)=\operatorname{trace} R T(S)=\langle R, T(S)\rangle .
\end{aligned}
$$

Finally we show that $T$ maps $\delta$ onto $\delta_{K}$. Indeed if $R \in \Re_{K}$ and $\langle R, T(S)\rangle=0$ for all $S \in \mathcal{S}$, then $0=\langle R, T(S)\rangle=\langle T(R), S\rangle=\langle R, S\rangle$ for all $S \in \mathcal{S}$ implying that $R \in \mathscr{B}$ and hence $R \in \mathscr{B}_{K}$. Conversely, the same argument shows that if $R \in \mathscr{B}_{K}$ then $\langle R, T(S)\rangle=0$ for all $S \in \mathcal{S}$.

2. Dimension four. In this section, for dimension $V=4$, we give a characterization of positive holomorphic sectional curvature and describe the minimum and maximum sets of $\sigma$, the holomorphic sectional curvature function.

For dimension $V=4$, the Hodge star operator $*: \Lambda^{2}(V) \rightarrow \Lambda^{2}(V)$ is an element of $\mathcal{R}$. It is known that $*$ generates $\mathcal{S}$ and that $\xi \in \Lambda^{2}(V)$ is decomposable if and only if $\langle * \xi, \xi\rangle=0$ (see Singer and Thorpe [3]). It follows that $T(*)$ generates $\mathcal{S}_{K}$ and that $\xi \in \Lambda^{2}(V)^{J}$ is decomposable if and only if $\langle T(*) \xi, \xi\rangle=0$.

Let $\Re_{K}^{+}=\left\{R \in \Re_{K} \mid\langle R \xi, \xi\rangle \geqslant 0\right.$ for all $\left.\xi \in \Lambda^{2}(V)\right\}$ and $\mathscr{B}_{K}^{+}=\{R \in$ $\mathscr{B}_{K} \mid \sigma_{R}(\xi)=\langle R \xi, \xi\rangle \geqslant 0$ for all $\left.\xi \in G^{J}\right\}$. We define $\pi$ as an orthogonal projection from $\mathscr{R}_{K}$ into $\mathscr{B}_{K}$. If $R=B+S, R \in \mathscr{R}_{K}, B \in \mathscr{B}_{K}$ and $S \in \mathcal{S}_{K}$, then $\sigma_{R}=\sigma_{B}$, and it follows that $\pi\left(R_{K}^{+}\right) \subseteq \mathscr{B}_{K}^{+}$.

TheOrem. If dimension $V=4$, then the map $\pi: \Re_{K}^{+} \rightarrow \mathscr{B}_{K}^{+}$is onto.

Corollary. Let dimension $V=4$ and $R \in \Re_{K}$ with $\sigma_{R}>0$ and $Z(R) \neq \varnothing$. Then there exists a unique $S \subseteq \mathcal{S}_{K}$ such that $Z(R)=G^{J} \cap \operatorname{Ker}(R+S)$.

Proof. Clearly we can find $S_{0} \in \mathcal{S}_{K}$ such that $R+S_{0}=B$ with $B \in \mathscr{B}_{K}^{+}$. Applying the theorem to $B$ we obtain $S_{1} \in \mathcal{S}_{K}$ such that $B+S_{1} \in R_{K}^{+}$. Let $S=S_{0}+S_{1}$; then $R+S \in R_{K}^{+}$. Thus $Z(R)=Z(R+S)=G^{J} \cap \operatorname{Ker}(R+S)$. To establish uniqueness, suppose that $S, S^{\prime} \in \mathcal{S}_{K}$ satisfy the conclusion of the corollary. Then for $\xi \in Z(R),(R+S) \xi=0$ and $\left(R+S^{\prime}\right) \xi=0$, and so $\left(S-S^{\prime}\right) \xi$ $=0$. But $S-S^{\prime} \in \mathcal{\delta}_{K}$ and hence must be a multiple of $T(*)$ which is nonsingular in $\Lambda^{2}(V)^{J}$. Thus $S=S^{\prime}$. 
COROllary. Let dimension $V=4$ and $R \in \Re_{K}$, and let $\lambda$ denote the minimum (or maximum) value of $\sigma_{R}$. Then there exists a unique $S \in \delta_{K}$ such that

$$
\left\{\xi \in G^{J} \mid \sigma_{R}(\xi)=\lambda\right\}=G \cap \operatorname{Ker}(R-\lambda T(I)+S) .
$$

Proof. It follows immediately from the previous corollary, replacing $\boldsymbol{R}$ by $R-\lambda T(I)$ (or by $\lambda T(I)-R$ if $\lambda$ is a maximum).

To prove the Theorem we will need the following lemma.

Lemma. For $\xi \in \Lambda^{2}(V)^{J}$ set $g(\xi)=\langle T(*) \xi, \xi\rangle$. Let $M=\{\xi \mid g(\xi)>0\}$ and $N=$ $\{\xi \mid g(\xi)<0\}$. For $R \in \mathscr{R}_{K}$ set $Q=\{\xi \mid\langle R \xi, \xi\rangle<0\}$. If $\sigma_{R} \geqslant 0$, then $Q \subseteq M$ or $Q \subseteq N$.

Proof. Let $S=\left\{\xi \in \Lambda^{2}(V)^{J}|| \xi \mid=1\right\}$. Note that $\xi \in S$ belongs to $G^{J}$ if and only if $g(\xi)=0$. If $Q \cap M \neq \varnothing$ then the function $\xi \rightarrow\langle R \xi, \xi\rangle$ assumes a minimum on $M \cap S$. In order to show this we consider the sets $S_{i}=\{\xi \in S \mid g(\xi)>$ $1 / i\}$. Each $S_{i}$ is a compact subset of $S, S_{i} \supseteq S_{i-1}$ and $\cup_{i=1}^{\infty} S_{i}=M \cap S$. Now, on each compact set $S_{i}$ the continuous function $\xi \rightarrow\langle R \xi, \xi\rangle$ assumes a minimum at some point $\xi_{i} \in S_{i}$. Since $Q \cap M \neq \varnothing$ there exists $\eta \in M \cap S$ such that $\langle R \eta, \eta\rangle=c<0$. If we assume the function $\xi \rightarrow\langle R \xi, \xi\rangle$ does not attain a minimum on $M \cap S$ then the sequence $\left\{\xi_{i}\right\}$ contains a subsequence, which we abusively denote $\left\{\xi_{i}\right\}$, with the properties that $\xi_{i} \in S_{i}-S_{i-1}$ and $\left\langle R \xi_{i}, \xi_{i}\right\rangle<$ $\left\langle R \xi_{i-1}, \xi_{i-1}\right\rangle\left\langle c\right.$. But then $\lim _{i \rightarrow \infty} g\left(\xi_{i}\right)=0$ and $\left\langle R \xi_{i}, \xi_{i}\right\rangle\langle c$ for all $i$ which contradicts $\sigma_{R} \geqslant 0$.

The minimum value $\mu_{0}<0$ is an eigenvalue (apply the technique of Lagrange multipliers to the function $\xi \rightarrow\langle R \xi$, $\xi\rangle$ on $S$ ) and so there exists $\xi_{0} \in M \cap S$ such that $R \xi_{0}=\mu_{0} \xi_{0}$.

Similarly if $Q \cap N \neq \varnothing$, then there exists $\xi_{1} \in N \cap S$ such that $R \xi_{1}=\mu_{1} \xi_{1}$ where $\mu_{1}<0$.

Clearly $R$ is negative definite on the span of $\left\{\xi_{0}, \xi_{1}\right\}$. But $g\left(\xi_{0}\right)>0$ and $g\left(\xi_{1}\right)<0$ implies that $g\left(t \xi_{0}+(1-t) \xi_{1}\right)=0$ for some $0<t<1$. This contradicts $\sigma_{R}>0$.

Proof of The Theorem. Consider $R_{t}=R+t T(*), t$ a real number, and set $Q_{t}=\left\{\xi \in \Lambda^{2}(V)^{J} \mid\left\langle R_{t} \xi, \xi\right\rangle<0\right\}$. It is not hard to see that for sufficiently large $t$, $Q_{t} \subseteq N$ and that for sufficiently negative $t, Q_{t} \subseteq M$ (in fact for each $t, Q_{t} \subseteq M$ or $\left.Q_{t} \subseteq N\right)$. Thus we can define $b=\sup \left\{t \mid Q_{t} \subseteq M\right\}$ and $a=\inf \left\{t \mid Q_{t} \subseteq N\right\}$. It is easily checked that $a \leqslant b$. For $t \in[a, b], Q_{t} \subseteq M$ and $Q_{t} \subseteq N$, and so $Q_{t}=\varnothing$. We thus conclude that for each $t \in[a, b], R+t T(*)$ is positive semidefinite. To complete the proof we set $S=t_{0} T(*), t_{0} \in[a, b]$.

3. Higher dimensions. In this section, for each $n \geqslant 3$, we will construct a Kähler curvature operator with nonnegative holomorphic sectional curvature whose zero set is not the intersection of $G^{J}$ with a linear subspace of $\Lambda^{2}(V)$.

First, we order the orthonormal basis for $\Lambda^{2}(V)^{J}$ (see $\S 1$ ) as follows:

$$
\begin{gathered}
e_{1} \wedge e_{1^{*}}, e_{2} \wedge e_{2^{*}}, e_{3} \wedge e_{3^{*}}, \quad\left(e_{1} \wedge e_{2}+e_{1^{*}} \wedge e_{2^{*}}\right) / \sqrt{2}, \\
\left(e_{1} \wedge e_{3}+e_{1^{*}} \wedge e_{3^{*}}\right) / \sqrt{2}, \quad\left(e_{2} \wedge e_{3}+e_{2^{*}} \wedge e_{3^{*}}\right) / \sqrt{2}, \ldots
\end{gathered}
$$


The curvature operator $R$, with respect to this basis, is defined by the symmetric matrix

$$
\left[\begin{array}{c|c|c}
A & 0 & 0 \\
\hline 0 & 0 & 0 \\
\hline 0 & 0 & 2 I
\end{array}\right]
$$

where $A$ is the matrix

$$
\left[\begin{array}{rrr}
1 & -1 & -1 \\
-1 & 1 & -1 \\
-1 & -1 & 1
\end{array}\right]
$$

and the central block is the $3 \times 3$ zero matrix. Observe that the matrix $A$ has eigenvalues $2,2,-1$.

For $R$ restricted to the span of $e_{1} \wedge e_{1^{*}}, e_{2} \wedge e_{2^{*}}$ and $e_{3} \wedge e_{3^{*}}$ let $a, b$ and $c$ be an orthonormal basis of eigenvectors with eigenvalues 2,2 and -1 respectively. We denote by $e, f$ and $g$ the vectors $\left(e_{1} \wedge e_{2}+e_{1^{*}} \wedge e_{2 *}\right) / \sqrt{2}$, $\left(e_{1} \wedge e_{3}+e_{1^{*}} \wedge e_{3^{*}}\right) / \sqrt{2}$ and $\left(e_{2} \wedge e_{3}+e_{2^{*}} \wedge e_{3^{*}}\right) / \sqrt{2}$, which are a basis for the kernel of $R$, and by $h_{i}$ the remaining vectors in the orthonormal basis for $\Lambda^{2}(V)^{J}$. An arbitrary vector $\xi \in \Lambda^{2}(V)^{J}$ can be written

$$
\xi=\alpha a+\beta b+\gamma c+\delta e+\varepsilon f+\rho g+\sum_{i} \lambda_{i} h_{i}
$$

Thus

$$
\langle R \xi, \xi\rangle=2 \alpha^{2}+2 \beta^{2}-\gamma^{2}+2 \sum_{i} \lambda_{i}^{2}
$$

Assuming that $|\xi|=1$, we have

$$
\langle R \xi, \xi\rangle=2-3 \gamma^{2}-2 \delta^{2}-2 \varepsilon^{2}-2 \rho^{2} \text {. }
$$

Now, suppose that $\xi \in G^{J}$. We can write $\xi=x \wedge J x$ with $x=\Sigma_{i} x_{i} e_{i}+\Sigma_{i} x_{i^{*}} e_{i^{*}}$ and $|x|=1$. Then

$$
\delta=\sqrt{2}\left(x_{1^{*}} x_{2}-x_{1} x_{2^{*}}\right), \quad \varepsilon=\sqrt{2}\left(x_{1 *} x_{3}-x_{1} x_{3^{*}}\right), \quad \rho=\sqrt{2}\left(x_{2^{*}} x_{3}-x_{2} x_{3^{*}}\right) .
$$

It is easy to see that $c=\left(e_{1} \wedge e_{1^{*}}+e_{2} \wedge e_{2^{*}}+e_{3} \wedge e_{3^{*}}\right) / \sqrt{3}$, thus

$$
\gamma=\xi \cdot c=\frac{1}{\sqrt{3}}\left(x_{1}^{2}+x_{1^{*}}^{2}+x_{2}^{2}+x_{2^{*}}^{2}+x_{3}^{2}+x_{3^{*}}^{2}\right) \text {. }
$$

After substitution we obtain the following formula for the holomorphic sectional curvature of $R$ :

$$
\begin{aligned}
\sigma_{R}(\xi)= & \langle R \xi, \xi\rangle=2-\left(x_{1}^{2}+x_{1^{*}}^{2}+x_{2}^{2}+x_{2^{*}}^{2}+x_{3}^{2}+x_{3^{*}}^{2}\right)^{2}-4\left(x_{1^{*}} x_{2}-x_{1} x_{2^{*}}\right)^{2} \\
& -4\left(x_{1^{*}} x_{3}-x_{1} x_{3^{*}}\right)^{2}-4\left(x_{2^{*}} x_{3}-x_{2^{*}} x_{3^{*}}\right)^{2} .
\end{aligned}
$$

Next, we will show that $\langle R \xi, \xi\rangle \geqslant 0$. Let $z=\left(x_{1}, x_{2}, x_{3}\right)$ and $z^{*}=$ $\left(x_{1^{*}}, x_{2^{*}}, x_{3^{*}}\right)$. Then the last three terms in the formula for $\langle R \xi, \xi\rangle$ are precisely $-4\left|z \times z^{*}\right|^{2}$, where $\times$ denotes the standard cross-product in euclidean 3-space. 
Since $\left|z \times z^{*}\right| \leqslant|z|\left|z^{*}\right|$ we have

$$
\begin{aligned}
\sigma_{R}(\xi)> & 2-\left(x_{1}^{2}+x_{1^{*}}^{2}+x_{2}^{2}+x_{2^{*}}^{2}+x_{3}^{2}+x_{3^{*}}^{2}\right)^{2} \\
& -4\left(x_{1}^{2}+x_{2}^{2}+x_{3}^{2}\right)\left(x_{1^{*}}^{2}+x_{2^{*}}^{2}+x_{3^{*}}^{2}\right) .
\end{aligned}
$$

Let $|z|^{2}=x_{1}^{2}+x_{2}^{2}+x_{3}^{2}=s$ and $\left|z^{*}\right|^{2}=x_{1^{*}}^{2}+x_{2^{*}}^{2}+x_{3^{*}}^{2}=t$; then $\sigma_{R}(\xi)>2$ $-(s+t)^{2}-4 s t$. Since $0 \leqslant s+t \leqslant 1$, we have

$$
2-(s+t)^{2}-4 s t \geqslant 2(s+t)^{2}-(s+t)^{2}-4 s t=(s-t)^{2}>0 .
$$

From the computations above we see that $\sigma_{R}(\xi)=0$ if and only if $\left|z \times z^{*}\right|=$ $|z|\left|z^{*}\right|$ and $|z|^{2}=\left|z^{*}\right|^{2}=\frac{1}{2}$. Thus the sectional curvature is zero at $\xi \in G^{J}$ if and only if $\xi=x \wedge J x$ with $x_{i}=x_{i^{*}}=0, i \geqslant 4, x_{1} x_{1^{*}}+x_{2} x_{2^{*}}+x_{3} x_{3^{*}}=0$ and $x_{1}^{2}+$ $x_{2}^{2}+x_{3}^{2}=x_{1^{*}}^{2}+x_{2^{*}}^{2}+x_{3^{*}}^{2}=\frac{1}{2}$. These conditions allow us to parametrize the zero set. First we note that by rotating $x$ in the plane spanned by $x$ and $J x$ we can reduce $x_{1}$ to 0 . Since $\left(x_{1^{*}}, x_{2^{*}}, x_{3^{*}}\right)$ is perpendicular to $\left(0, x_{2}, x_{3}\right)$ we can write $\left(x_{1^{*}}, x_{2^{*}}, x_{3^{*}}\right)=\cos \theta(1 / \sqrt{2}, 0,0)+\sin \theta\left(0,-x_{3}, x_{2}\right)$. Therefore we have the parametrization

$$
\begin{array}{ll}
x_{1}=0, & x_{1^{*}}=\frac{1}{\sqrt{2}} \cos \theta, \\
x_{2}=\frac{1}{\sqrt{2}} \sin \phi, & x_{2^{*}}=-\frac{1}{\sqrt{2}} \sin \theta \cos \phi, \\
x_{3}=\frac{1}{\sqrt{2}} \cos \phi, & x_{3^{*}}=\frac{1}{\sqrt{2}} \sin \theta \sin \phi, \\
x_{i}=0, & x_{i^{*}}=0, \quad i>4 .
\end{array}
$$

Hence any zero of $\sigma_{R}$ must have the form

$$
\begin{aligned}
\xi= & \frac{1}{2} \cos ^{2} \theta\left(e_{1} \wedge e_{1^{*}}\right)+\frac{1}{2}\left(\sin ^{2} \phi+\cos ^{2} \phi \sin ^{2} \theta\right)\left(e_{2} \wedge e_{2^{*}}\right) \\
& +\frac{1}{2}\left(\cos ^{2} \phi+\sin ^{2} \phi \sin ^{2} \theta\right)\left(e_{3} \wedge e_{3^{*}}\right)+\frac{1}{2} \sin \phi \cos \theta\left(e_{1} \wedge e_{2}+e_{1^{*}} \wedge e_{2^{*}}\right) \\
& +\frac{1}{2} \cos \phi \cos \theta\left(e_{1} \wedge e_{3}+e_{1^{*}} \wedge e_{3^{*}}\right)-\frac{1}{2} \sin \theta\left(e_{2} \wedge e_{3}+e_{2^{*}} \wedge e_{3^{*}}\right) \\
& -\frac{1}{2} \cos \phi \sin \theta \cos \theta\left(e_{1} \wedge e_{2^{*}}-e_{1^{*}} \wedge e_{2}\right) \\
& +\frac{1}{2} \sin \phi \sin \theta \cos \theta\left(e_{1} \wedge e_{3^{*}}-e_{1^{*}} \wedge e_{3}\right) \\
& +\frac{1}{2} \sin \phi \cos \phi \cos ^{2} \theta\left(e_{2} \wedge e_{3^{*}}-e_{2^{*}} \wedge e_{3}\right) .
\end{aligned}
$$

Taking different values for $\phi$ and $\theta$ it is easy to see that the linear span of the zero set is the linear subspace of $\Lambda^{2}(V)^{J}$ generated by the vectors which appear in the formula for $\xi$ above. This linear span clearly contains vectors for which the sectional curvature is not zero e.g. $e_{1} \wedge e_{1^{*}}$.

To obtain an example satisfying the Bianchi identity we use the projection $\pi$ : $\Re_{K} \rightarrow \mathscr{B}_{K}$ defined in $\$ 2$. As it was mentioned before, this projection does not change the holomorphic sectional curvature.

Finally, we remark that our example shows that the Theorem in $\$ 2$ does not extend to higher dimensions. 


\section{REFERENCES}

1. D. L. Johnson, Sectional curvature and curvature normal forms, Michigan Math. J. 27 (1980), 275-294.

2. A. M. Naveira and C. Fuertes, The zeroes of nonnegative holomorphic curvature operators, Trans. Amer. Math. Soc. 213 (1975), 139-147.

3. I. M. Singer and J. A. Thorpe, The curvature of 4-dimensional Einstein spaces, Global Analysis, Univ. of Tokyo Press, Tokyo; Princeton Univ. Press, Princeton, N.J., 1969, pp. 355-364.

4. J. A. Thorpe, On the curvature tensor of a positively curved 4-manifold, Proc. 13th Biennial Seminar Canad. Math. Congress (1971).

5. S. M. Zoltek, Nonnegative curvature operators: some nontrivial examples, J. Differential Geom. 14 (1979), 303-315.

Department of Mathematics, University of Georgia, Athens, Georgla 30602

Department of Mathematical Sciences, George Mason University, Fairfax, Virginia 22030 